\title{
Dual-Hop OFDM Opportunistic AF Relaying Under Joint Transmit/Receive I/Q Imbalance
}

\author{
M. Mokhtar ${ }^{\dagger}$, A.-A. A. Boulogeorgos*, G. K. Karagiannidis* and N. Al-Dhahir ${ }^{\dagger}$ \\ ${ }^{\dagger}$ Department of Electrical Engineering and Computer Science, University of Texas at Dallas, USA, Emails \{m.mokhtar, aldhahir $\} @$ utdallas.edu \\ *Department of Electrical and Computer Engineering, Aristotle University of Thessaloniki, Greece, Emails: \{ampoulog, geokarag\}@auth.gr
}

\begin{abstract}
We study the outage performance of orthogonal frequency-division multiplexing dual-hop opportunistic amplifyand-forward relaying in the presence of $I / Q$ imbalance in all of the nodes' transceivers. We derive a closed-form expression for the end-to-end outage probability for the general case, where each node suffers from a different $I / Q$ imbalance level and under the assumption that the distance between source and relay is not the same for all relays. Our simulation results show excellent match to our analysis and both demonstrate that uncompensated I/Q imbalance can be detrimental but it can also be effectively mitigated using a few relays. This result has a significant impact on relay deployment in future wireless communication networks.
\end{abstract}

Index Terms-I/Q imbalance, Amplify-and-forward relay, Opportunistic Relaying, OFDM

\section{INTRODUCTION}

Wireless networks with cooperative relaying have received considerable attention recently and have been adopted in wireless standards such as LTE [1]. The performance of amplify-and-forward (AF)-based relaying has been analyzed in several papers (see [2] and reference therein), assuming an ideal radio frequency (RF) front-end. However, in practice, the system performance is degraded by RF impairments, such as high-power amplifier (HPA) nonlinearity, in-phase and quadrature-phase (I/Q) imbalance (IQI), low-noise amplifier (LNA) nonlinearity, antenna coupling, phase noise (PN) and carrier frequency offset (CFO) [3]- [4]. In particular, IQI represents the mismatch between analog components in the I and Q branches, which results from the limited accuracy of analog hardware and it can be either frequency independent or frequency dependent. Frequency independent IQI occurs mainly due to non-ideal mixers and phase shifters and is constant over the whole signal bandwidth, while frequencydependent IQI is due to I and Q low-pass filters mismatches. For orthogonal frequency-division multiplexing (OFDM) systems, IQI degrades the system performance severely due to mirror subcarrier interference [5].

Scanning the literature, the impact of PN on the performance of OFDM-based AF relay networks was investigated in [4], where it was shown that the use of an AF-relay is not beneficial compared to a direct transmission, as the PN level exceeds a certain threshold. Furthermore, the outage performance for the AF relaying protocol was studied in [3] and [6]. Specifically, in [3] IQI was considered only at the destination and flat fading links were assumed, while in [6]

The work of M. Mokhtar and N. Al-Dhahir was supported by QNRF. both the relay and the destination suffer from IQI in the presence of frequency-selective fading links.

In this paper, we investigate the effect of IQI in all nodes of an OFDM dual-hop opportunistic relaying system with multiple AF relays. Specifically, we derive a closed-form expression for the end-to-end outage probability for the general case, where each node suffers from a different IQI level and without restricting the source-relay distance to be the same for all relays. To gain more insight, the special case where all relays lie on the perpendicular line midway between the source $(S)$ and destination $(D)$ and all nodes experience the same IQI level is investigated in detail. In this case, it is shown that the outage probability floor is proportional to the product of the signal constellation size and the IQI signal to interference ratio raised to a power equal to the number of relays.

The rest of this paper is organized as follow. The system model is described in Section II and the outage probability is analyzed in Section III followed by simulation results in Section IV to validate our analysis. Finally, the paper is concluded in Section V.

Notations: Unless otherwise stated, lower and upper case bold letters denote vectors and matrices, respectively. The matrices $\mathbf{I}$ and $\mathbf{F}$ denote, respectively, the identity matrix and the Fast Fourier transform (FFT) matrix whose middle row corresponds to the DC, and their subscripts denote their size. For matrices, $\mathbf{A}^{\sharp} \triangleq \tilde{\mathbf{I}}_{M} \mathbf{A}^{*} \tilde{\mathbf{I}}_{M}$, while for vectors, $\mathbf{a}^{\sharp} \triangleq \tilde{\mathbf{I}}_{M} \mathbf{a}^{*}$ is the reversal (image) permutation matrix. Also, $(\cdot)^{H},(\cdot)^{*}$, $(\cdot)^{T}$ denote the matrix complex-conjugate transpose, complexconjugate and transpose operations, respectively. The operators $\mathbb{E}[\cdot]$ and $|\cdot|$ denote the statistical expectation and the absolute value, respectively.

\section{SySTEM MODEL}

We consider the downlink transmission scenario in an $\mathrm{AF}$ opportunistic relaying system where the source, $J$ relays and destination nodes are equipped with a single antenna. OFDM transmission is used in each of the two time slots to combat the channel's frequency selectivity. In the first slot, $S$ transmits to the relay node $(R)$, while in the second slot, one relay is selected to amplify and forward the received signal to $D$, while the source and the other relays remain silent.

The fading channels of all the links are assumed independent, frequency-selective and fixed over at least one OFDM symbol duration. The RF front-ends of the $S, R$ and $D$ nodes are assumed to be impaired with different IQI levels. The $R$ node suffers from both receive and transmit IQI. We consider 
frequency-independent IQI caused by the gain and the phase mismatches between the I and the Q branches denoted by $\epsilon_{x}^{t / r}$ and $\theta_{x}^{t / r}$, respectively, where the subscript $x$ is the node identifier $(S, R$ or $D)$ and the superscript $t / r$ denotes the up/down-conversion process, respectively. The time-domain (TD) baseband IQI-impaired signal is given by $g_{I Q I}^{t / r}(t)$, where

$$
g_{I Q I}^{t / r}(t)=\mu_{x}^{t / r} g(t)+v_{x}^{t / r} g^{*}(t) .
$$

with

$$
\mu_{x}^{t / r}=\cos \left(\frac{\theta_{x}^{t / r}}{2}\right) \mp j \epsilon_{x}^{t / r} \sin \left(\frac{\theta_{x}^{t / r}}{2}\right),
$$

and the minus, plus signs denote the up and down-conversion processes, respectively,

$$
v_{x}^{t / r}=\epsilon_{x}^{t / r} \cos \left(\frac{\theta_{x}^{t / r}}{2}\right)-j \sin \left(\frac{\theta_{x}^{t / r}}{2}\right) .
$$

Note that $g(t)$ is the TD baseband IQI-free signal and the $g^{*}(t)$ term arises due to the IQI effect.

Assuming that $\overline{\mathbf{s}}$ is the TD $M \times 1$ data vector with $\mathbb{E}\left[\overline{\mathbf{s}} \overline{\mathbf{s}}^{H}\right]=\eta_{o} \mathbf{I}_{M}$, the source transmits

$$
\overline{\mathbf{s}}_{S}=\mu_{S}^{t} \overline{\mathbf{s}}+v_{S}^{t} \overline{\mathbf{s}}^{*} .
$$

The TD received signals in the first time slot at the $i^{\text {th }} R$ node after removing the cyclic-prefix $(\mathrm{CP})$ is given by

$$
\begin{aligned}
\overline{\mathbf{r}}_{R}^{(i)}= & \mu_{R^{(i)}}^{r} \overline{\mathbf{H}}_{S R^{(i)}} \overline{\mathbf{s}}_{S}+v_{R^{(i)}}^{r}\left(\overline{\mathbf{H}}_{S R^{(i)}} \overline{\mathbf{s}}_{S}\right)^{*}+ \\
& \mu_{R^{(i)}}^{r} \overline{\mathbf{n}}_{R^{(i)}}+v_{R^{(i)}}^{r} \overline{\mathbf{n}}_{R^{(i)}}^{*},
\end{aligned}
$$

where $R^{(i)}$ denotes the $i^{\text {th }} R$ node. The $M \times M$ circulant channel matrix $\overline{\mathbf{H}}_{U V}$ models the channel from node $U$ to $V$ and its first column is $\left[\bar{h}_{U V}^{T} 0_{1 \times M-L}\right]^{T}$, where $\bar{h}_{U V}$ is the channel impulse response (CIR) with $L$ complex Gaussian taps. Moreover, $\overline{\mathbf{n}}_{R^{(i)}}$ is the circularly symmetric complex additive white Gaussian noise (AWGN) vectors with singlesided power spectrum densities of $N_{0}$.

From (4), $\overline{\mathbf{r}}_{R}^{(i)}$ can be written as

$$
\begin{aligned}
\overline{\mathbf{r}}_{R}^{(i)}= & \left(\mu_{R^{(i)}}^{r} \overline{\mathbf{H}}_{S R^{(i)}} \mu_{S}^{t}+v_{R^{(i)}}^{r} \overline{\mathbf{H}}_{S R^{(i)}}^{*}\left(v_{S}^{t}\right)^{*}\right) \overline{\mathbf{s}}+ \\
& \left(\mu_{R^{(i)}}^{r} \overline{\mathbf{H}}_{S R^{(i)}} v_{S}^{t}+v_{R^{(i)}}^{r} \overline{\mathbf{H}}_{S R^{(i)}}^{*}\left(\mu_{S}^{t}\right)^{*}\right) \overline{\mathbf{s}}^{*}+ \\
& \mu_{R^{(i)}}^{r} \overline{\mathbf{n}}_{R^{(i)}}+v_{R^{(i)}}^{r} \overline{\mathbf{n}}_{R^{(i)}}^{*} .
\end{aligned}
$$

In the second time slot, the relay amplifies the received signal and forwards it to the destination. To keep the complexity low at the relay, the channel is not estimated at the relay. Hence, the amplification factor is defined as

$$
\alpha=\sqrt{\frac{\eta_{1}}{\mathbb{E}\left[\left|\overline{\mathbf{r}}_{R}^{(i)}\right|^{2}\right]}},
$$

where $\eta_{1}$ is the average energy transmitted by the $R^{(i)}$. Therefore, the TD baseband equivalent signal transmitted by $R^{(i)}$ in the second time-slot is given by

$$
\overline{\mathbf{s}}_{R}^{(i)}=\alpha\left\{\mu_{R^{(i)}}^{t} \overline{\mathbf{r}}_{R^{(i)}}+v_{R^{(i)}}^{T} \overline{\mathbf{r}}_{R^{(i)}}^{*}\right\} .
$$

From (6), $\bar{s}_{R}$ can be written as shown in (9), given at the top of the next page.

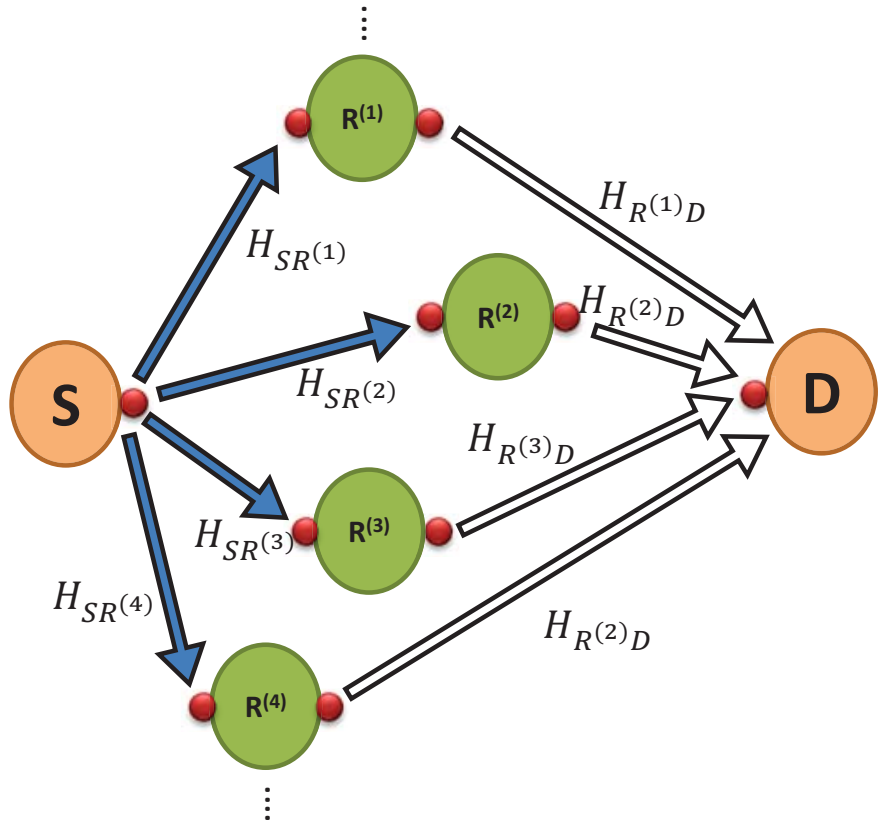

Fig. 1. System model block diagram where solid and hollow arrows show transmissions during the first and second time slots, respectively. The red bubbles indicate IQI, i.e. its presence on the RHS/LHS of the node means IQI at the Tx/Rx, respectively.

The TD baseband equivalent signal received at the $D$ node in the second time-slot is given by

$$
\overline{\mathbf{r}}_{D}=\mu_{D}^{r} \overline{\mathbf{H}}_{R^{(i)} D} \overline{\mathbf{s}}_{R^{(i)}}+v_{D}^{r}\left(\overline{\mathbf{H}}_{R^{(i)} D} \bar{s}_{R^{(i)}}\right)^{*}+\mu_{D}^{r} \overline{\mathbf{n}}_{D}+v_{D}^{r} \overline{\mathbf{n}}_{D}^{*} .
$$

From (9), $\overline{\mathbf{r}}_{D}$ can be written as

$$
\overline{\mathbf{r}}_{D}=\left(\mu_{D}^{r} \overline{\mathbf{H}}_{1}+v_{D}^{r} \overline{\mathbf{H}}_{2}\right) \bar{s}+\left(v_{D}^{r} \overline{\mathbf{H}}_{1}^{*}+\mu_{D}^{r} \overline{\mathbf{H}}_{2}^{*}\right) \overline{\mathbf{s}}^{*}+\overline{\mathbf{z}},
$$

where

$$
\begin{aligned}
& \overline{\mathbf{H}}_{1}=\alpha \overline{\mathbf{H}}_{R^{(i)} D} \\
& \left\{\mu_{R^{(i)}}^{t}\left[\mu_{R^{(i)}}^{r} \overline{\mathbf{H}}_{S R^{(i)}} \mu_{S}^{t}+v_{R^{(i)}}^{r} \overline{\mathbf{H}}_{S R^{(i)}}^{*}\left(v_{S}^{t}\right)^{*}\right]\right. \\
& \left.+v_{R^{(i)}}^{t}\left[\left(\mu_{R^{(i)}}^{r}\right)^{*} \overline{\mathbf{H}}_{S R^{(i)}}^{*}\left(v_{S}^{t}\right)^{*}+\left(v_{R^{(i)}}^{r}\right)^{*} \overline{\mathbf{H}}_{S R^{(i)}} \mu_{S}^{t}\right]\right\}, \\
& \overline{\mathbf{H}}_{2}=\alpha \overline{\mathbf{H}}_{R^{(i)} D}^{*} \\
& \left\{\left(\mu_{R^{(i)}}^{t}\right)^{*}\left[\left(\mu_{R^{(i)}}^{r}\right)^{*} \overline{\mathbf{H}}_{S R^{(i)}}^{*}\left(v_{S}^{t}\right)^{*}+\left(v_{R^{(i)}}^{r}\right)^{*} \overline{\mathbf{H}}_{S R^{(i)}}\left(\mu_{S}^{t}\right)\right]\right. \\
& \left.+\left(v_{R^{(i)}}^{t}\right)^{*}\left[\mu_{R^{(i)}}^{r} \overline{\mathbf{H}}_{S R^{(i)}} \mu_{S}^{t}+v_{R^{(i)}}^{r} \overline{\mathbf{H}}_{S R^{(i)}}^{*}\left(v_{S}^{t}\right)^{*}\right]\right\},
\end{aligned}
$$

and

$$
\begin{aligned}
& \overline{\mathbf{z}}=\mu_{D^{r}}^{r} \overline{\mathbf{H}}_{R^{(i)} D} \alpha\left\{\mu_{R^{(i)}}^{t}\left[\mu_{R^{(i)}}^{r} \overline{\mathbf{n}}_{R}+v_{R^{(i)}}^{r} \overline{\mathbf{n}}_{R^{(i)}}^{*}\right]\right. \\
& \left.+v_{R^{(i)}}^{t}\left[\left(\mu_{R^{(i)}}^{r}\right)^{*} \overline{\mathbf{n}}_{R^{(i)}}^{*}+\left(v_{R^{(i)}}^{r}\right)^{*} \overline{\mathbf{n}}_{R^{(i)}}\right]\right\} \\
& +v_{R^{(i)}}^{r} \overline{\mathbf{H}}_{R^{(i) D}}^{*} \alpha\left\{\left(\mu_{R^{(i)}}^{t}\right)^{*}\left[\left(\mu_{R^{(i)}}^{r}\right)^{*} \overline{\mathbf{n}}_{R^{(i)}}^{*}+\left(v_{R^{(i)}}^{r}\right)^{*} \overline{\mathbf{n}}_{R^{(i)}}\right]\right. \\
& \left.+\left(v_{R^{(i)}}^{t}\right)^{*}\left[\mu_{R^{(i)}}^{r} \overline{\mathbf{n}}_{R^{(i)}}+v_{R^{(i)}}^{r} \overline{\mathbf{n}}_{R^{(i)}}^{*}\right]\right\}+\mu_{D}^{r} \overline{\mathbf{n}}_{D}+v_{D}^{r} \overline{\mathbf{n}}_{D}^{*} .
\end{aligned}
$$




$$
\begin{aligned}
\overline{\mathbf{s}}_{R}^{(i)} & =\alpha\left\{\mu_{R^{(i)}}^{t}\left[\mu_{R^{(i)}}^{r} \overline{\mathbf{H}}_{S R^{(i)}} \mu_{S}^{t}+v_{R^{(i)}}^{r} \overline{\mathbf{H}}_{S R^{(i)}}^{*}\left(v_{s}^{t}\right)^{*}\right]+v_{R^{(i)}}^{t}\left[\left(\mu_{R^{(i)}}^{r}\right)^{*} \overline{\mathbf{H}}_{S R^{(i)}}^{*}\left(v_{S}^{t}\right)^{*}+\mu_{s}^{t} \overline{\mathbf{H}}_{S R^{(i)}}\left(v_{R^{(i)}}^{r}\right)^{*}\right]\right\} \overline{\mathbf{s}} \\
& +\alpha\left\{\mu_{R^{(i)}}^{t}\left[\mu_{R^{(i)}}^{r} \overline{\mathbf{H}}_{S R^{(i)}} v_{S}^{t}+v_{R^{(i)}}^{r} \overline{\mathbf{H}}_{S R^{(i)}}^{*}\left(\mu_{S}^{t}\right)^{*}\right]+v_{R^{(i)}}^{t}\left[\left(\mu_{R^{(i)}}^{r}\right)^{*} \overline{\mathbf{H}}_{S R^{(i)}}^{*}\left(\mu_{S}^{t}\right)^{*}+\left(v_{R^{(i)}}^{r}\right)^{*} \overline{\mathbf{H}}_{S R^{(i)}} v_{S}^{t}\right]\right\} \overline{\mathbf{s}}^{*} \\
& +\alpha \mu_{R^{(i)}}^{t}\left[\mu_{R^{(i)}}^{r} \overline{\mathbf{n}}_{R^{(i)}}+v_{R^{(i)}}^{r} \overline{\mathbf{n}}_{R^{(i)}}^{*}\right]+\alpha v_{R^{(i)}}^{t}\left[\left(\mu_{R^{(i)}}^{r}\right)^{*} \overline{\mathbf{n}}_{R^{(i)}}^{*}+\left(v_{R^{(i)}}^{r}\right)^{*} \overline{\mathbf{n}}_{R^{(i)}}\right]
\end{aligned}
$$

Based on the above model, the frequency-domain (FD) received signal at the $D$ node in the second slot is obtained by taking the FFT of (11), and can be written as follows

$$
\mathbf{r}_{D}=\left(\mu_{D}^{r} \mathbf{H}_{1}+v_{D}^{r} \mathbf{H}_{2}\right) \mathbf{s}+\left(v_{D}^{r} \mathbf{H}_{1}^{\sharp}+\mu_{D}^{r} \mathbf{H}_{2}^{\sharp}\right) \mathbf{s}^{\sharp}+\mathbf{z} .
$$

In (15), $\mathbf{H}_{1}$ and $\mathbf{H}_{2}$ can be easily obtained from (12) and (13) by replacing each $\overline{\mathbf{H}}_{U V}$ and $\overline{\mathbf{H}}_{U V}^{*}$ with $\mathbf{H}_{U V}$ and $\mathbf{H}_{U V}^{\sharp}$, respectively, where $\mathbf{H}_{U V}$ is the $M \times M$ diagonal matrix whose diagonal elements are the channel frequency response (CFR) coefficients from node $U$ to $V$ and $\mathbf{H}_{U V}(k)$ is the $k^{\text {th }}$ element on the main diagonal of matrix $\mathbf{H}_{U V}$.

\section{Performance Analysis}

In this section, we investigate the effect of IQI on the outage performance. The end-to-end outage probability is defined as the probability of the equivalent output signal-to-interferenceplus-noise (SINR) falling below a given threshold.

According to the opportunistic relaying (OR) scenario, the participating selected relay is the one with the highest SINR $\gamma_{A F}^{(i)}(k)$ which be written as follows

$$
\gamma_{A F}(k)=\max _{i \in\{1, \ldots, J\}} \gamma_{A F}^{(i)}(k),
$$

where $\gamma_{A F}^{(i)}(k)$ is given by [4],

$$
\begin{aligned}
\gamma_{A F}^{(i)}(k) & =\frac{\gamma_{S R}^{(i)}(k) \gamma_{R D}^{(i)}(k)}{\gamma_{S R}^{(i)}(k)+\gamma_{R D}^{(i)}(k)+1} \\
& <\frac{\gamma_{S R}^{(i)}(k) \gamma_{R D}^{(i)}(k)}{\gamma_{S R}^{(i)}(k)+\gamma_{R D}^{(i)}(k)} \\
& \leq \min \left(\gamma_{S R}^{(i)}(k), \gamma_{R D}^{(i)}(k)\right),
\end{aligned}
$$

where $\gamma_{S R}^{(i)}(k)$ and $\gamma_{R D}^{(i)}(k)$ denote the SINR between $S \rightarrow$ $R^{(i)}$ and $R^{(i)} \rightarrow D$ links, respectively, and they can be evaluated from Eqs. (18) and (19) (given in the next page). Hence, the end-to-end outage probability is given by [7]

$$
\begin{aligned}
P_{A F} & =P_{r}\left(\log _{2}\left(1+\gamma_{A F}(k)\right)<r_{\mathrm{th}}\right) \\
& =P_{r}\left(\gamma_{A F}(k)<2^{r_{\mathrm{th}}}-1\right) \\
& =\prod_{i=1}^{J} P_{A F^{(i)}}\left(\gamma_{A F}^{(i)}(k)<2^{r_{\mathrm{th}}}-1\right),
\end{aligned}
$$

where $r_{\text {th }}$ is double the required rate ${ }^{1}$ and $P_{A F^{(i)}}\left(\gamma_{A F}^{(i)}(k)<u\right)$ is the cumulative density function $(\mathrm{CDF})$ of $\gamma_{A F}^{(i)}(k)$ which can be written as

$$
\begin{aligned}
& P_{A F^{(i)}}\left(\gamma_{A F}^{(i)}(k)<2^{r_{\mathrm{th}}}-1\right)= \\
& 1-\left[1-P_{r}\left(\gamma_{S R}^{(i)}(k) \leq \gamma_{\mathrm{th}}\right)\right]\left[1-P_{r}\left(\gamma_{R D}^{(i)}(k) \leq \gamma_{\mathrm{th}}\right)\right],
\end{aligned}
$$

\footnotetext{
${ }^{1}$ Because the communication occurs over two time slot.
}

where $P_{r}\left(\gamma_{S R}^{(i)}(k) \leq \gamma_{\text {th }}\right)$ and $P_{r}\left(\gamma_{R D}^{(i)}(k) \leq \gamma_{\text {th }}\right)$ are the CDFs of $\gamma_{S R}^{(i)}(k)$ and $\gamma_{R D}^{(i)}(k)$, respectively, and $\gamma_{\text {th }}=2^{r_{\text {th }}}-1$. To evaluate the CDF of $\gamma_{S R}^{(i)}$, we re-write (18) as follows

$$
\gamma_{S R}^{(i)}(k) \equiv \frac{\left|\tilde{Z}_{S R^{(i)}}\right|^{2}}{\left|\tilde{W}_{S R^{(i)}}\right|^{2}+N_{S R^{(i)}}} \equiv \frac{Z_{S R^{(i)}}}{W_{S R^{(i)}}+N_{S R^{(i)}}} .
$$

where

$$
\begin{aligned}
& \tilde{Z}_{S R^{(i)}}=a_{S R^{(i)}}^{(i)} \mathbf{H}_{S R^{(i)}}(k)+b_{S R^{(i)}}^{(i)} \mathbf{H}_{S R^{(i)}}^{*}(-k), \\
& \tilde{W}_{S R^{(i)}}=c_{S R^{(i)}}^{(i)} \mathbf{H}_{S R^{(i)}}(k)+d_{S R^{(i)}}^{(i)} \mathbf{H}_{S R^{(i)}}^{*}(-k)
\end{aligned}
$$

and

$$
N_{S R^{(i)}}=\frac{N_{0}}{\eta_{o}}\left(\left|\mu_{R^{(i)}}^{r}\right|^{2}+\left|v_{R^{(i)}}^{r}\right|^{2}\right),
$$

with $a_{S R^{(i)}}^{(i)}=\mu_{R^{(i)}}^{r} \mu_{S}^{t}, b_{S R^{(i)}}^{(i)}=v_{R^{(i)}}^{r}\left(v_{S}^{t}\right)^{*}, c_{S R^{(i)}}^{(i)}=$ $\mu_{R^{(i)}}^{r} v_{S}^{t}$ and $d_{S R^{(i)}}^{(i)}=v_{R^{(i)}}^{r}\left(\mu_{S}^{t}\right)^{*}$.

To simplify $\gamma_{S R}^{(i)}(k)$, we take into account that for practical values of IQI levels $b_{S R^{(i)}}^{(i)} \simeq 0$. Moreover, except for few central subcarriers (near DC), the correlation between the $k^{\text {th }}$ subcarrier and its image is small due to their large spectral separation, and therefore, they can considered independent ${ }^{2}$. Therefore,

$$
2 \mathbb{E}\left[\Re\left\{c_{1} d_{1}^{*} \mathbf{H}_{S R^{(i)}}^{*}(k) \mathbf{H}_{S R^{(i)}}^{*}(-k)\right\}\right] \simeq 0 .
$$

Hence, we can approximate $Z_{S R^{(i)}}$ and $W_{S R^{(i)}}$ as follows [8]

$$
Z_{S R^{(i)}} \simeq\left|a_{S R^{(i)}}^{(i)}\right|^{2}\left|\mathbf{H}_{S R^{(i)}}(k)\right|^{2}
$$

and

$$
\begin{aligned}
W_{S R^{(i)}} & =\left|c_{S R^{(i)}}^{(i)} \mathbf{H}_{S R^{(i)}}(k)+d_{S R^{(i)}}^{(i)} \mathbf{H}_{S R^{(i)}}^{*}(-k)\right|^{2} \\
& \simeq\left|c_{S R^{(i)}}^{(i)} \mathbf{H}_{S R^{(i)}}(k)\right|^{2}+\left|d_{S R^{(i)}}^{(i)} \mathbf{H}_{S R^{(i)}}^{*}(-k)\right|^{2}
\end{aligned}
$$

where $\left|\mathbf{H}_{U V}(k)\right|^{2}$ is an exponentially-distributed random variable with mean $\lambda_{U V}^{k}$ proportional to $\left(d_{U V}\right)^{-\xi}$ where $d_{U V}$ is the distance between nodes $U$ and $V$ and $\xi$ is the pathloss exponent.

Substituting (27), (28) into (22), we can accurately approximate $\gamma_{S R}^{(i)}(k)$ as

$$
\begin{aligned}
& \gamma_{S R}^{(i)}(k) \simeq \\
& \frac{\left|a_{S R^{(i)}}^{(i)}\right|^{2}\left|H_{S R^{(i)}}(k)\right|^{2}}{\left|c_{S R^{(i)}}^{(i)} H_{S R^{(i)}}(k)\right|^{2}+\left|d_{S R^{(i)}}^{(i)} H_{S R^{(i)}}^{*}(-k)\right|^{2}+N_{S R^{(i)}}} \\
& \triangleq \frac{a_{S R^{(i)} X}}{c_{S R^{(i)} X} X+d_{S R}^{(i)} Y+N_{S R^{(i)}}}
\end{aligned}
$$

\footnotetext{
${ }^{2}$ Uncorrelated Gaussian random variables are independent.
} 


$$
\begin{aligned}
\gamma_{S R}^{(i)}(k) & =\frac{\left|\mu_{R^{(i)}}^{r} \mu_{S}^{t} \mathbf{H}_{S R^{(i)}}(k)+v_{R^{(i)}}^{r}\left(v_{S}^{t}\right)^{*} \mathbf{H}_{S R^{(i)}}^{*}(-k)\right|^{2}}{\left|\mu_{R^{(i)}}^{r} v_{S}^{t} \mathbf{H}_{S R^{(i)}}(k)+v_{R^{(i)}}^{r}\left(\mu_{S}^{t}\right)^{*} \mathbf{H}_{S R^{(i)}}^{*}(-k)\right|^{2}+\frac{N_{0}}{\eta_{o}}\left(\left|\mu_{R^{(i)}}^{r}\right|^{2}+\left|v_{R^{(i)}}^{r}\right|^{2}\right)} \\
\gamma_{R D}^{(i)}(k) & =\frac{\left|\mu_{D}^{r} \mu_{R^{(i)}}^{t} \mathbf{H}_{R^{(i)} D}(k)+v_{D}^{r}\left(v_{R^{(i)}}^{t}\right)^{*} \mathbf{H}_{R^{(i)} D}^{*}(-k)\right|^{2}}{\left|\mu_{D}^{r} v_{R^{(i)}}^{t} \mathbf{H}_{R^{(i)} D}(k)+v_{D}^{r}\left(\mu_{R^{(i)}}^{t}\right)^{*} \mathbf{H}_{R^{(i)} D}^{*}(-k)\right|^{2}+\frac{N_{0}\left(\left|\mu_{D}^{r}\right|^{2}+\left|v_{D}^{r}\right|^{2}\right)}{\eta_{1}\left|\mu_{R^{(i)}}^{r}\right|^{2}}}
\end{aligned}
$$

with $a_{S R}^{(i)}=\left|a_{S R^{(i)}}^{(i)}\right|^{2}, c_{S R}^{(i)}=\left|c_{S R^{(i)}}^{(i)}\right|^{2}, d_{S R}^{(i)}=\left|d_{S R^{(i)}}^{(i)}\right|^{2}$ and $X, Y$ are exponentially-distributed random variables with probability density functions (PDFs)

$$
f_{X}(x)=\frac{1}{\lambda_{S R^{(i)}}^{k}} e^{-\frac{x}{\lambda_{S R^{(i)}}^{k}}}, f_{Y}(y)=\frac{1}{\lambda_{S R^{(i)}}^{-k}} e^{-\frac{y}{\lambda_{S R^{(i)}}^{-k}}} .
$$

Exploiting the independence between $X$ and $Y$, we evaluate the CDF of $\gamma_{S R}^{(i)}(k)$ as follows [7]

$P_{r}\left(\gamma_{S R}^{(i)}(k) \leq \gamma_{\text {th }}\right)$

$= \begin{cases}\int_{0}^{\infty} P_{X}\left(x \leq \frac{\gamma_{\mathrm{th}}\left(d_{S R}^{(i)} y+N_{S R}^{(i)}\right)}{a_{S R}^{(i)}-\gamma_{\mathrm{th}} c_{S R}^{(i)}}\right) P_{Y}(y) d y, & \gamma_{\mathrm{th}} \leq \frac{a_{S R}^{(i)}}{c_{S R}^{(i)}} \\ 1, & \text { O.W. }{ }^{3}\end{cases}$

Carrying out the integration in (31), we get

$$
\begin{aligned}
& P_{r}\left(\gamma_{S R}^{(i)}(k) \leq \gamma_{\mathrm{th}}\right) \\
& =1-\frac{\lambda_{S R^{(i)}}^{k}\left(a_{S R}^{(i)}-\gamma_{\mathrm{th}} c_{S R}^{(i)}\right)}{\lambda_{S R^{(i)}}^{k}\left(a_{S R}^{(i)}-\gamma_{\mathrm{th}} c_{S R}^{(i)}\right)+d_{S R}^{(i)} \gamma_{\mathrm{th}} \lambda_{S R^{(i)}}^{-k}} \\
& \quad \times e^{-\frac{N_{S R^{(i)}} \gamma_{\mathrm{th}}}{\left.\left(a_{S R}^{(i)} \gamma_{\mathrm{th}} c_{S R}^{(i)}\right) \lambda_{S R}^{k}{ }^{(i)}\right)}}, \gamma_{\mathrm{th}} \leq \frac{a_{S R}^{(i)}}{c_{S R}^{(i)}} .
\end{aligned}
$$

Moreover, assuming uncorrelated scattering, $\lambda_{S R^{(i)}}^{k}=$ $\lambda_{S R^{(i)}}^{-k}=\lambda_{S R^{(i)}}$, and Eq. (32) can be simplified further as

$$
\begin{aligned}
& P_{r}\left(\gamma_{S R}^{(i)}(k) \leq \gamma_{\mathrm{th}}\right)=1-\frac{\left(a_{S R}^{(i)}-\gamma_{\mathrm{th}} c_{S R}^{(i)}\right)}{\left(a_{S R}^{(i)}-\gamma_{\mathrm{th}} c_{S R}^{(i)}\right)+d_{S R}^{(i)} \gamma_{\mathrm{th}}} \\
& \times e^{-\frac{N_{S R^{(i)}} \gamma_{\mathrm{th}}}{\left(a_{S R}^{(i)}-\gamma_{\mathrm{th}} c_{S R}^{(i)}\right) \lambda_{S R}(i)}}, \gamma_{\mathrm{th}} \leq \frac{a_{S R}^{(i)}}{c_{S R}^{(i)}} .
\end{aligned}
$$

Similarly,

$$
\begin{aligned}
& P_{r}\left(\gamma_{R D}^{(i)}(k) \leq \gamma_{\mathrm{th}}\right)=1-\frac{\left(a_{R D}^{(i)}-\gamma_{\mathrm{th}} c_{R D}^{(i)}\right)}{\left(a_{R D}^{(i)}-\gamma_{\mathrm{th}} c_{R D}^{(i)}\right)+d_{R D}^{(i)} \gamma_{\mathrm{th}}} \\
& \times e^{-\frac{N_{R^{(i)}} \gamma_{\mathrm{th}}}{\left(a_{R D}^{(i)}-\gamma_{\mathrm{th}} c_{R D}^{(i)}\right) \lambda_{R^{(i)}}}}, \gamma_{\mathrm{th}} \leq \frac{a_{R D}^{(i)}}{c_{R D}^{(i)}},
\end{aligned}
$$

where $a_{R D}^{(i)}=\left|a_{R^{(i)} D}^{(i)}\right|^{2}=\left|\mu_{D}^{r} \mu_{R^{(i)}}^{t}\right|^{2}, c_{R D}^{(i)}=\left|c_{R^{(i)} D}^{(i)}\right|^{2}=$ $\left|\mu_{D}^{r} v_{R^{(i)}}^{t}\right|^{2}, \quad d_{R D}^{(i)}=\left|d_{R^{(i)} D}^{(i)}\right|^{2}=\left|v_{D}^{r}\left(\mu_{R^{(i)}}^{t}\right)^{*}\right|^{2}$ and $N_{R^{(i)} D}=\frac{N_{0}\left(\left|\mu_{D}^{r}\right|^{2}+\left|v_{D}^{r}\right|^{2} \mid\right)}{\eta_{1}\left|\mu_{R^{(i)}}^{r}\right|^{2}}$.

\footnotetext{
${ }^{3}$ Henceforth, for notational convenience, the condition on $\gamma_{t h}$ such that the outage probability becomes one is omitted.
}

Substituting Eqs. (33) and (34) into (21) to derive $P_{A F^{(i)}} \forall i$ and then substituting the result into (20), we get

$P_{A F}=$

$\prod_{i \in \mathcal{W}}\left(1-\frac{\left(a_{S R}^{(i)}-\gamma_{\mathrm{th}} c_{S R}^{(i)}\right)\left(a_{R D}^{(i)}-\gamma_{\mathrm{th}} c_{R D}^{(i)}\right)}{\left[\left(a_{S R}^{(i)}-\gamma_{\mathrm{th}} c_{S R}^{(i)}\right)+d_{S R}^{(i)} \gamma_{\mathrm{th}}\right]\left[\left(a_{R D}^{(i)}-\gamma_{\mathrm{th}} c_{R D}^{(i)}\right)+d_{R D}^{(i)} \gamma_{\mathrm{th}}\right]}\right.$

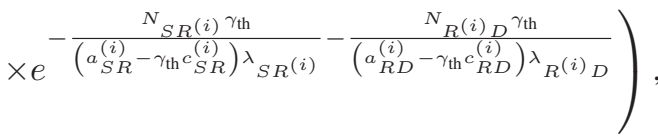

where $\mathcal{W}=\left\{i: i \in\{1, \cdots, J\} \& \gamma_{\text {th }} \leq \min \left\{\frac{a_{S R}^{(i)}}{c_{S R}^{(i)}}, \frac{a_{R D}^{(i)}}{c_{R D}^{(i)}}\right\}\right\}$.

\section{An Insightful Special Case}

Next, to gain more insight, we investigate a special case, where all nodes suffer from the same IQI level, $\mathcal{W}=$ $\{1, \cdots, J\}$, and the relay nodes lie on the perpendicular line midway between the $S$ and $D$ nodes. In this case Eq. (35) reduces to

$$
P_{A F}=\prod_{i=1}^{J}\left(1-\left(\frac{1}{1+\eta}\right)^{2} e^{-\frac{\left(N_{S R^{(i)}}+N_{R^{(i) D}}\right) \gamma_{\mathrm{th}}}{\left(a-\gamma_{\mathrm{th}} c\right) \lambda(i)}}\right),
$$

where $\eta=\frac{c \gamma_{\mathrm{th}}}{-c \gamma_{\mathrm{th}}+a}, a=|\mu|^{4}, c=|\mu v|^{2}, \quad \lambda^{(i)}=$ $\lambda_{S R^{(i)}}=\lambda_{R^{(i)} D}$ for the $i^{\text {th }}$ relay node, and $\left(\mu_{S}^{t}\right)^{*}=\mu_{D}^{r}=$ $\mu_{R^{(i)}}^{r}=\left(\mu_{R^{(i)}}^{t}\right)^{*}=\mu$ and $\nu_{S}^{t}=\nu_{D}^{r}=\nu_{R^{(i)}}^{r}=\nu_{R^{(i)}}^{r}=$ $\nu, \forall i \in\{1, \cdots, J\}$. Moreover, in the high SNR region $\left(N_{S R^{(i)}} \rightarrow 0\right.$ and $\left.N_{R^{(i) D}} \rightarrow 0\right)$ the outage probability floor is given by

$$
P_{A F} \doteq\left(1-\left(\frac{1}{1+\eta}\right)^{2}\right)^{J}
$$

where $\doteq$ denotes the asymptotic equivalence at high SNR.

For practical IQI levels, $\eta<1$ and, hence, $\eta^{2}<<1$. Therefore, Eq. (37) can be approximated as

$$
\begin{aligned}
P_{A F} & \doteq\left(1-(1-\eta)^{2}\right)^{J}=\left(\eta^{2}+2 \eta\right)^{J} \\
& \simeq 2^{J} \eta^{J} \simeq 2^{J} \frac{c^{J} \gamma_{\text {th }}^{J}}{a^{J}} \\
& \simeq\left(2\left|\frac{\nu}{\mu}\right|^{2}\left(2^{r_{\text {th }}}-1\right)\right)^{J} .
\end{aligned}
$$

From Eq. (38), it is evident that the outage probability floor is proportional to $\left|\frac{\nu}{\mu}\right|^{2 J}$ for a certain signal constellation size, where $\left|\frac{\nu}{\mu}\right|^{2}$ represents the IQI interference-to-signal ratio 
which is typically $<<1$. Hence, the diversity effect of OR reduces the IQI outage floor significantly, even for few relays. On the other hand, the outage probability floor worsens as the signal constellation size increases due to the increased sensitivity of bigger signal constellation to IQI effects. Furthermore, Eq. (38) shows that the outage probability floor is independent of the relays' positions on the perpendicular line midway between $S$ and $D$.

\section{Numerical and Simulation Results}

In this section, we present Monte-Carlo simulation results to demonstrate the accuracy of the outage probability expressions derived in Section III. Although our analytical expressions are general for any AF relay network layout and IQI levels, we consider the following special but insightful scenario. In this scenario, unless otherwise stated, we assume three relays located on a line perpendicular to the line connecting the $\mathrm{S}$ and $\mathrm{D}$ nodes and we denote the distance between this line the source by $d_{1}$. In addition, the distance between the $S$ and $D$ nodes is normalized to $d_{S D}=1$; meanwhile, the distance between each pair of consecutive relays on the line is $d_{S D} / 2$.

The CFR between nodes $U$ and $V$ is generated using $L=8$ uncorrelated zero-mean complex Gaussian taps with uniform power-delay profile and the variance of each tap is assumed to be $\frac{d_{U V}^{-\xi}}{L}$, where $\xi$ is set to 3.7 . The source and the relay nodes transmit with the same power, $\eta_{0}=\eta_{i}=1$; hence, $N_{S R^{(i)}}=N_{R^{(i) D}}=\frac{1}{S N R}, \forall i$. Finally, the number of OFDM subcarriers is 64 and the subcarrier index of interest is 15 .

In Fig. 2, we assume that $d_{1}=0.5$, i.e. the relays lie on a perpendicular line between $S$ and $D$. Therefore, the second relay lies on the line connecting the $S$ and $D$ nodes, while the other two relays form isosceles triangles with them. Moreover, all nodes suffer from the same IQI levels, i.e. $\left(\mu_{S}^{t}\right)^{*}=\mu_{D}^{r}=$ $\mu_{R^{(i)}}^{r}=\left(\mu_{R^{(i)}}^{t}\right)^{*}$ and $\nu_{S}^{t}=\nu_{D}^{r}=\nu_{R^{(i)}}^{r}=\nu_{R^{(i)}}^{r} \forall i$. Fig. 2 depicts the outage probabilities for different IQI levels and $r_{\text {th }}=4 \mathrm{bits} / \mathrm{sec} / \mathrm{Hz}$ and it shows the accuracy of the derived expressions in (35). The outage probability simulations and analytical expressions coincide over the entire range of SNR for different IQI levels. Moreover, Eq. (38) accurately predicts the outage probability floor and illustrates its dependence on the IQI level.

Fig. 3 shows the impact of the number of relays on the outage probability floor for $d_{1}=0.5$ when all nodes are impaired by the same IQI level of $10 \log _{10}\left(1+\epsilon^{t / r}\right)=0.15$ $\mathrm{dB}$ and $\theta^{t / r}=5^{\circ}$. At low SNR, there is no performance improvement due to each additional relay because its pathloss is greater than the existing relays' pathlosses and; hence, it is less likely to be selected. On the other hand, at high SNR and as shown by (38), the outage probability floor is independent of pathloss and the relays' positions while being directly proportional to $\left|\frac{\nu}{\mu}\right|^{2 J}$. With 7 relays, the outage probability floor is almost eliminated for the considered SNR range.

For further assessment of the derived expressions, we show in Fig. 4 the outage probabilities for a different scenario where the source and the destination nodes suffer from the same IQI level with amplitude and phase mismatches of $0.25 \mathrm{~dB}$ and $5^{\circ}$, respectively, while the three relays are IQI-impaired with

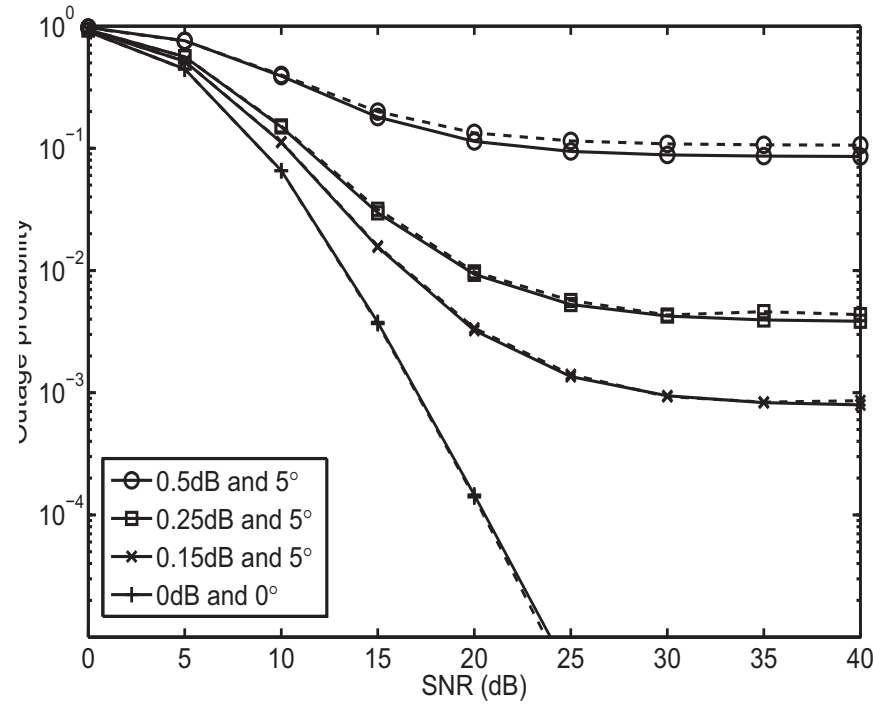

Fig. 2. Analytical (solid lines) and simulated (dashed lines) outage probabilities for different IQI levels and $r_{\text {th }}=4 \mathrm{bits} / \mathrm{sec} / \mathrm{Hz}$.

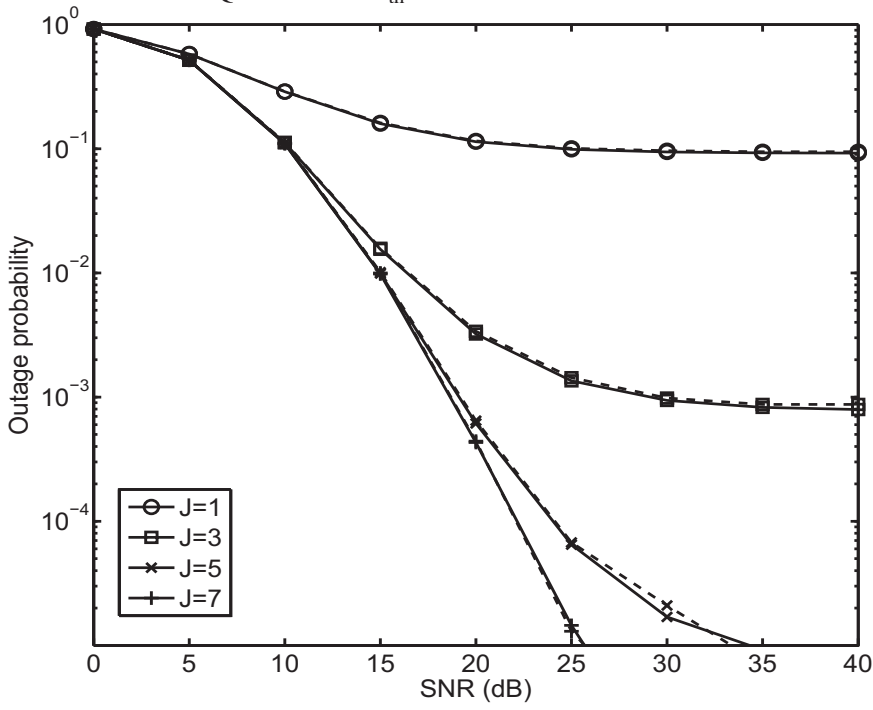

Fig. 3. Analytical (solid lines) and simulated (dashed lines) outage probabilities for IQI level of $0.15 \mathrm{~dB}$ and $5^{\circ}$ and $r_{\text {th }}=4 \mathrm{bits} / \mathrm{sec} / \mathrm{Hz}$.

the same phase mismatch of $5^{\circ}$ and amplitude mismatches of $0.1,0.15$, and $0.2 \mathrm{~dB}$. The results in this figure verify the accuracy of the derived expressions even if the nodes suffer from different IQI levels and the relays are not at an equal distance from the $S$ and $D$ nodes. Furthermore, it is worth mentioning that the outage probability floor is independent of the channel gain, which reflects the effect of the relative distances between the relays and the $S$ and $D$ nodes, since it scales the signal and the interference by the same amount.

Finally, in Fig. 5 we assume that the nodes involved in the first time slot transmissions have perfect RF ends while those involved in the second time slot transmissions are IQIimpaired. In other words, the source is IQI-free, the relays have IQI-free receiving RF-ends and IQI-impaired transmitting RFends, and the destination node suffers from IQI. This implicitly assumes that the relays use different RF-ends for receiving and transmitting where the former is IQI-free while the latter is 


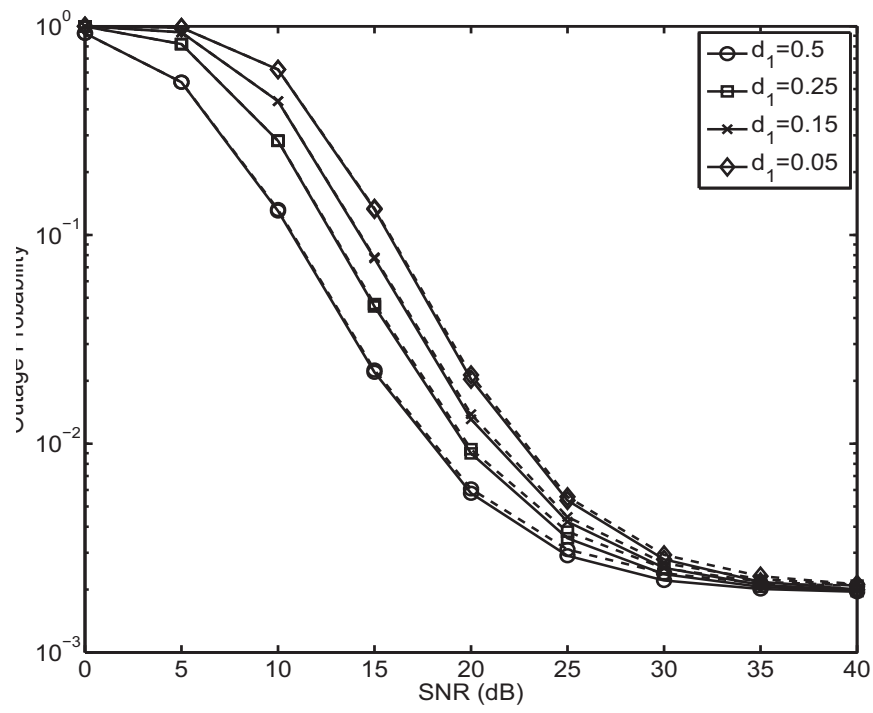

Fig. 4. Analytical (solid lines) and simulated (dashed lines) outage probabilities for $r_{\text {th }}=4 \mathrm{bits} / \mathrm{sec} / \mathrm{Hz}$.

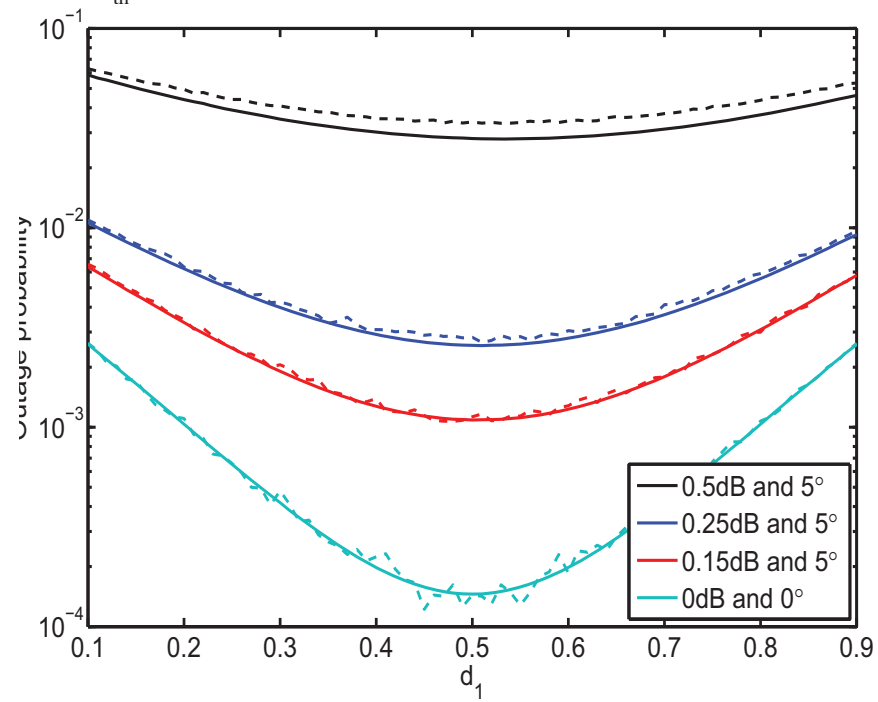

Fig. 5. Analytical (solid lines) and simulated (dashed lines) outage probabilities with $\mathrm{SNR}=20 \mathrm{~dB}$ and for $r_{\mathrm{th}}=4 \mathrm{bits} / \mathrm{sec} / \mathrm{Hz}$.

IQI-impaired. Although this is not a practical assumption since the relays use the same RF front-end for both transmitting and receiving, we make this assumption to validate our outage expressions. Moreover, we assume the same IQI levels at the relays and the destination nodes. As shown in Fig. 5, the simulations and the analytical expressions coincide over the whole range of $d_{1}$. It is well-known that the best position for the relays is to be in the middle between the source and the destination for the IQI-free scenario [9]. Interestingly, this is still best position even if the nodes suffer from IQI. However, as the IQI level increases, the sensitivity to the exact relay location decreases since the outage performance becomes dominated by IQI effects.

\section{CONCLUSiON}

In this paper, we studied the outage performance of OFDM AF opportunistic relaying in the presence of IQI in all nodes. We derived an accurate approximation for the outage probability for the general case where each node suffers from a different IQI level and without restricting the distance between the source and the relay to be the same for all relays. Our simulations illustrated the accuracy of the derived analytical expressions and illustrated that the detrimental IQI effects on outage can be significantly reduced using only few opportunistic relays. Finally, we considered a special but insightful scenario where all nodes suffer from the same IQI level and the relay nodes lie on the perpendicular line midway between the $S$ and $D$ nodes. In this case, we showed that the outage probability floor is proportional to the product of the signal constellation size and the IQI interference to signal ratio raised to a power equal to the number of relays.

\section{REFERENCES}

[1] C. Hoymann, W. Chen, J. Montojo, A. Golitschek, C. Koutsimanis, and $\mathrm{X}$. Shen, "Relaying operation in 3GPP LTE: challenges and solutions," IEEE Communications Magazine, no. 2, pp. 156-162, 2012.

[2] M. Dohler and Y. Li, Cooperative Communications: Hardware, Channel and PHY. Wiley, 2010.

[3] J. Qi, S. Aissa, and M.-S. Alouini, "Analysis and compensation of I/Q imbalance in amplify-and-forward cooperative systems," in IEEE Wireless Communications and Networking Conference (WCNC), 2012, pp. 215220.

[4] P. Rabiei, W. Namgoong, and N. Al-Dhahir, "On the Performance of OFDM-Based Amplify-and-Forward Relay Networks in the Presence of Phase Noise," IEEE Trans. on Comm., no. 5, pp. 1458 -1466, 2011.

[5] T. Schenk, RF Imperfections in High-Rate Wireless Systems. The Netherlands: Springer, 2008.

[6] M. Mokhtar, A. Gomaa, and N. Al-Dhahir, "OFDM AF Relaying Under I/Q Imbalance: Performance Analysis and Baseband Compensation," to appear in IEEE Transactions on Commumications, 2013.

[7] H. Stark and J. Woods, Probability, Statistics, and Random processes for engineers, 4th ed. Prentice Hall, 2012.

[8] B. Maham, O. Tirkkonen, and A. Hjorungnes, "Impact of Transceiver I/Q Imbalance on Transmit Diversity of Beamforming OFDM Systems," IEEE Transactions on Comm., vol. 60, no. 3, pp. 643-648, 2012.

[9] L. Fei, L. Qinghua, L. Tao, and Y. Guangxin, "Impact of Relay Location According to SER for Amplify-and-Forward Cooperative Communications," in IEEE International Workshop on Anti-counterfeiting, Security, Identification (ASID), 2007, pp. 324-327. 